\title{
OPEN Influence of heat flow control on dynamical spin injection in CoFeB/Pt/CoFeB trilayer
}

\author{
Sora Obinata, Riku limori, Kohei Ohnishi \& Takashi Kimura ${ }^{凶}$
}

A dynamical spin injection based on the ferromagnetic resonance in a ferromagnetic/nonmagnetic bi-layered structure, is a powerful mean for generating and manipulating the spin current. Although the mechanism of the dynamical spin injection is mainly attributed to the spin pumping, the detailed mechanism and the quantitative understanding for related phenomena are still controversial. As an another important contribution to the dynamical spin injection, the heating effect due to the resonant precessional motion of the magnetization is pointed out recently. In order to quantify the contribution from the heating effect, we here investigate the dynamical spin injection in a CoFeB/Pt/CoFeB trilayer. Although the contribution from the spin pumping diminishes because of the symmetric spin injection from the upper and lower interfaces, a significant inverse spin Hall voltage has been clearly observed. We show that the observed voltage can be quantitatively understood by the thermal spin injection due to a heating effect during the ferromagnetic resonance. A proper combination between the spin pumping and the heat-flow control in the multi-layered system is a key for the efficient dynamical spin injection.

Spintronic devices consisting of ferro-magnet (FM) /non-magnet (NM) hybrid structures have attracted much attention since the discovery of the spin-dependent transports ${ }^{1}$. It is well known that a spin current, a flow of spin angular momentum, plays a significant role in operations of the spintronic devices ${ }^{2}$. This is because the spin current can be utilized for manipulating the magnetization as well as for detecting the magnetization direction ${ }^{3,4}$. The spin current is, in general, created by applying an electric field across the FM/NM interface, resulting in injecting the spin-polarized electrons from the FM into the $\mathrm{NM}^{5-8}$. This is known as electrical spin injection, which is a widely utilized for generating the spin current because of its high selectivity and flexibility. In addition, since the Seebeck coefficient in the ferromagnet also shows the spin dependence, the spin current can be generated by using the temperature gradient across the FM/NM interface, instead of the electric field ${ }^{9-11}$. This is known as the thermal spin injection, where the generation efficiency often shows the significant enhancement in several ferromagnetic metals depending on its band structure ${ }^{12-14}$.

Apart from the aforementioned statical spin injection, the dynamical spin injection based on a ferromagnetic resonance (FMR) is also an attractive mean for generating and manipulating the spin current ${ }^{15-17}$. This is because the FMR can be induced by microwave irradiation without electrical connection, leading to wireless spintronics ${ }^{18,19}$. So far, the mechanism of the dynamical spin injection is attributed to the spin pumping, where the excess spins in the FM due to the FMR spill out into the adjacent nonmagnetic heavy metal (HM) $)^{20-22}$. On the other hand, the resonant precessional motion of the magnetization is known to yield the heating effect through the magnon-phonon interaction ${ }^{23-26}$. This FMR heating effect produces the temperature gradient across the FM/ $\mathrm{NM}$ interface, resulting in the thermal spin injection ${ }^{12,14}$. Since the temperature change due to the FMR heating exceeds $10 \mathrm{~K}$, it could be the significant contribution on the dynamical spin injection ${ }^{19,24}$. However, since the dynamical spin injection is mainly evaluated by measuring the inverse spin Hall effect (ISHE), it is difficult to distinguish the dominant contribution in the dynamical spin injection. Moreover, the proper combination between the spin pumping and the thermal spin injection will enhance the dynamical spin injection efficiency significantly. Therefore, it is essential to understand how large the thermal spin injection contributes to the dynamical spin injection. We have developed an effective device structure for the quantitative evaluation of the dynamical spin injection ${ }^{27}$. In this method, the spin-rectified voltage caused by the charge current directly flowing in the ferromagnetic layer can be minimized by using a thick $\mathrm{Cu}$ electrode, leading to the quantitative analysis of the dynamical spin injection. Very recently, we showed that a $\mathrm{CoFeB} / \mathrm{Pt} / \mathrm{CoFeB}$ trilayered structure is effective for the investigation of a dynamical spin injection ${ }^{28}$. In the present paper, by extending our evaluation method, 
(a)

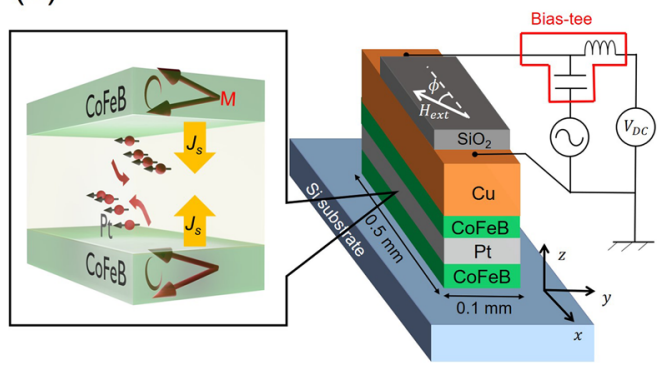

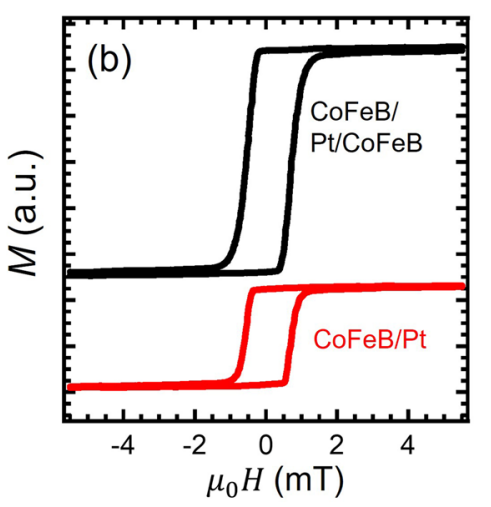

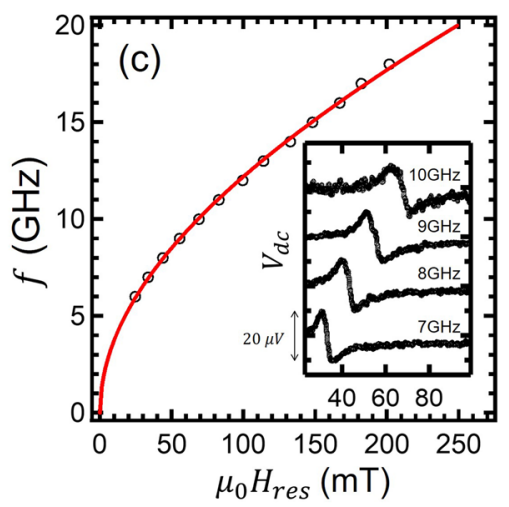

Figure 1. (a) Schematic illustration of the fabricated device based on $\mathrm{CoFeB} / \mathrm{Pt} / \mathrm{CoFeB}$ trilayer together with the probe configuration for RF measurements. The inset shows a conceptual image of the dynamical spin injection in the trilayer. (b) Room temperature $\mathrm{M}-\mathrm{H}$ curves measured by VSM for $\mathrm{CoFeB} / \mathrm{Pt}$ bilayer and $\mathrm{CoFeB} /$ $\mathrm{Pt} / \mathrm{CoFeB}$ trilayer. (c) Relationship between the resonant magnetic field $H_{\text {res }}$ and the microwave frequency $f$. The solid line represents the fitting curve using Kittel's equation. The inset shows the field dependence of dc voltage under the microwave signal with various frequencies observed in the trilayered sample.

we investigate the contribution of the thermal spin injection in dynamical spin injection. Especially, by controlling the temperature gradient due to FMR heating effect, we clarify the importance of the thermal spin injection.

\section{Experimental procedure}

The sample used for the present study consist of a $\mathrm{CoFeB} / \mathrm{Pt} / \mathrm{CoFeB}$ tri-layered structure with a $\mathrm{Cu}$ electrode, as schematically shown in Fig. 1a. Here, the trilayer has been grown by a magnetron sputtering system with the base pressure of $8 \times 10^{-6} \mathrm{~Pa}$ on a non-doped floating-zone Si substrate. The thickness of the Pt layer (the upper and bottom CoFeB layers) is $5 \mathrm{~nm}(10 \mathrm{~nm})$. Then, a 100-nm-thick $\mathrm{Cu}$ electrode was entirely deposited on the trilayer. Here, the multi-layered film was patterned to $100-\mu \mathrm{m}$-width and $500-\mu \mathrm{m}$-length rectangular-strip shape by the lithography. In order to avoid the surface oxidation, a 10-nm-thick $\mathrm{SiO}_{2}$ capping layer was sputtered on the top of the $\mathrm{Cu}$ electrode.

The present $\mathrm{CoFeB} / \mathrm{Pt} / \mathrm{CoFeB}$ tri-layered structure is effective for acquiring the contribution of the thermal spin injection selectively because of the following reason. When the upper and lower CoFeB layers have the same magnetic property, the FMR can be excited simultaneously. In such a situation, the dynamical spin injections occur both from the upper $\mathrm{CoFeB} / \mathrm{Pt}$ and lower $\mathrm{Pt} / \mathrm{CoFeB}$ interfaces ${ }^{15-17,19}$. As schematically shown in Fig. 1a, the flowing direction of the spin current injected from the upper and lower interfaces are opposite each other while the polarizations are the same direction. Since the spin pumping is the interfacial effect ${ }^{20-22}$, the magnitude of the spin injection due to the spin pumping from both sides are almost same. Therefore, the contribution of the spin pumping is expected to diminish in the present trilayer. On the other hand, from the viewpoint of the thermal spin injection, since the magnetic trilayer is sandwiched by the Cu electrode and the Si substrate, the temperature distribution becomes asymmetric ${ }^{12,14}$. In this case, the magnitude of the thermal spin injections from the upper and lower interfaces should be different. This leads to generate the detectable ISHE voltage. Thus, we are able to extract the contribution of the thermal spin injection due to the FMR heating effect effectively.

\section{Results and discussions}

First, we evaluate the static magnetic property of the trilayed film by using a vibrating-sample magnetometer (VSM). As show in Fig. 1b, the magnetization curve exhibits a single sharp switching with the coercivity as low as $5 \mathrm{Oe}$. For the comparison, we also show the magnetization curve for the $\mathrm{CoFeB} / \mathrm{Pt}$ bilayer. We confirm the magnetization curve similarly to the trilayed film with almost the same coercivity. These results indicate that the upper and lower $\mathrm{CoFeB}$ films have the same magnetic property with the typical characteristic of soft magnetic materials.

We then evaluate the dynamic property of the present trilayer by measuring the dc voltage along the longitudinal direction under the RF current injection, as schematically shown in Fig. 1a. Here, the microwave power is $-5 \mathrm{dBm}$ and the microwave frequency range is varied from $6 \mathrm{GHz}$ to $18 \mathrm{GHz}$. The inset of Fig. 1c shows the representative spectra observed in the present sample for various frequencies measured at an angle $\phi=45$ deg with respect to the current direction. The shapes of the spectra are almost asymmetric, suggesting that the ISHE signal, which produces the symmetric signal, is canceled out by the dynamical spin injections from both interfaces. The origin of the asymmetric is mainly due to the combination between the eddy current and the anisotropic magnetoresistance. Figure 1c shows the resonant magnetic field as a function of the microwave frequency. This dependence was confirmed to be well explained by Kittel's equation given by

$$
f_{0}=\frac{\gamma}{2 \pi} \sqrt{\mu_{0} H_{\text {res }}\left(\mu_{0} H_{\text {res }}+M_{S}\right)}
$$



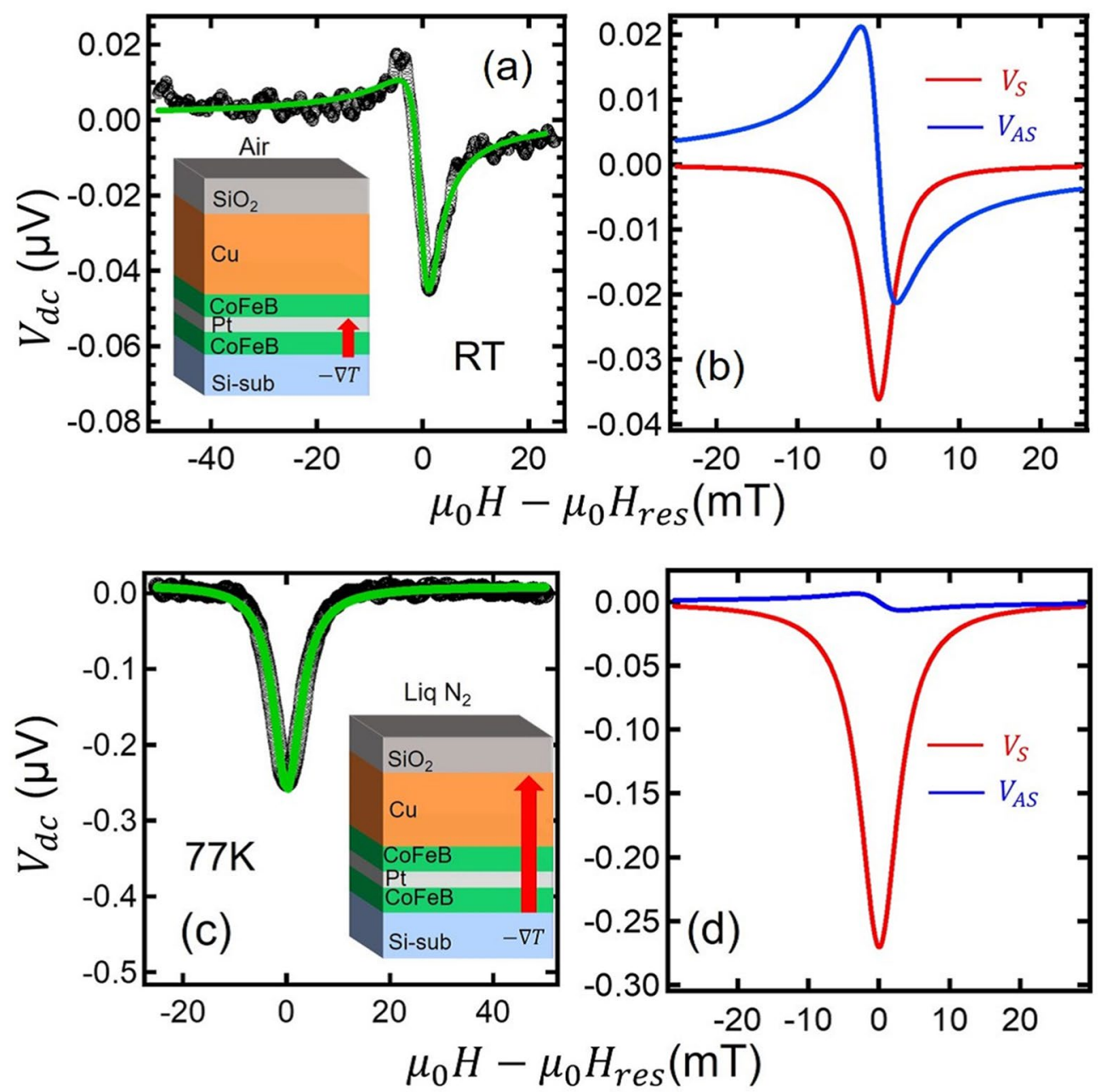

Figure 2. Field dependence of the dc voltage under the microwave with $f=6 \mathrm{GHz}$ measured at (a) room temperature and (c) $77 \mathrm{~K}$. The solid lines represent the fitting curve using Eq . (2). Separation of the fitting curve into symmetric (red) and anti-symmetric (blue) components for (b) room temperature and (d) $77 \mathrm{~K}$.

with the gyromagnetic ratio $\gamma / 2 \pi=28 \mathrm{GHz} / \mathrm{T}$ and the saturation magnetization $M_{S}=1.80 \mathrm{~T}$. This means that the observed signal is caused by the FMR of the CoFeB film ${ }^{29}$.

We now discuss the shape of the voltage spectra. Figure 2 a shows the voltage spectra with $f=6 \mathrm{GHz}$. Although the shape of the spectra is almost asymmetric, a small symmetric component has be confirmed. In order to analyze the spectra more quantitatively, we use the following equation ${ }^{30,31}$.

$$
V_{\text {tot }}\left(H_{\text {ext }}\right)=V_{\text {sym }} \frac{\Delta H^{2}}{\left(H_{\text {ext }}-H_{\text {res }}\right)^{2}+\Delta H^{2}}+V_{\text {asym }} \frac{\Delta H\left(H_{\text {ext }}-H_{\text {res }}\right)}{\left(H_{\text {ext }}-H_{\text {res }}\right)^{2}+\Delta H^{2}}
$$

Here, $V_{\text {sym }}$ and $V_{\text {asym }}$ are, respectively, the amplitude of the symmetric and anti-symmetric components and $\Delta H$ is the linewidth of the FMR peak. As seen in Fig. 2b, the fitting curve well reproduces the observed spectra and $V_{\text {sym }}$ and $V_{\text {asym }}$ are estimated as $-0.037 \mu \mathrm{V}$ and $0.021 \mu \mathrm{V}$, respectively. Thus, non-negligible symmetric component with the negative sign is obtained. This is because we did not apply the temperature control of the sample intentionally. According to the previous study ${ }^{19,27,28}$, the negative ISHE signal in the present coordinate corresponds to the dynamical spin injection from the upper interface. This indicates that the dynamical spin injection from the upper interface is larger than that from the lower interface. The small amplitude of the symmetric component is due to the cancellation of the dynamical spin injection from both side. We also observed the anti-symmetric field dependence within the same order of the magnitude. As mentioned in the introduction ${ }^{28}$, the origin of the anti-symmetric component is mainly caused by the galvanomagnetic effect due to the direct current flow in the FM, but its quantitative understanding is still controversial issue. Moreover, the similar undesired effects is known to contribute to the symmetric component. However, its magnitude is comparable or smaller than the anti-symmetric component. We emphasize that the clear negative change is observed in the symmetric signal even by subtracting the undesired component. This is a reasonable scenario because the generated heat mainly diffuses from the top to the bottom layers ( $-z$ direction) owing to the large thermal sink of the Si substrate. 
(a)
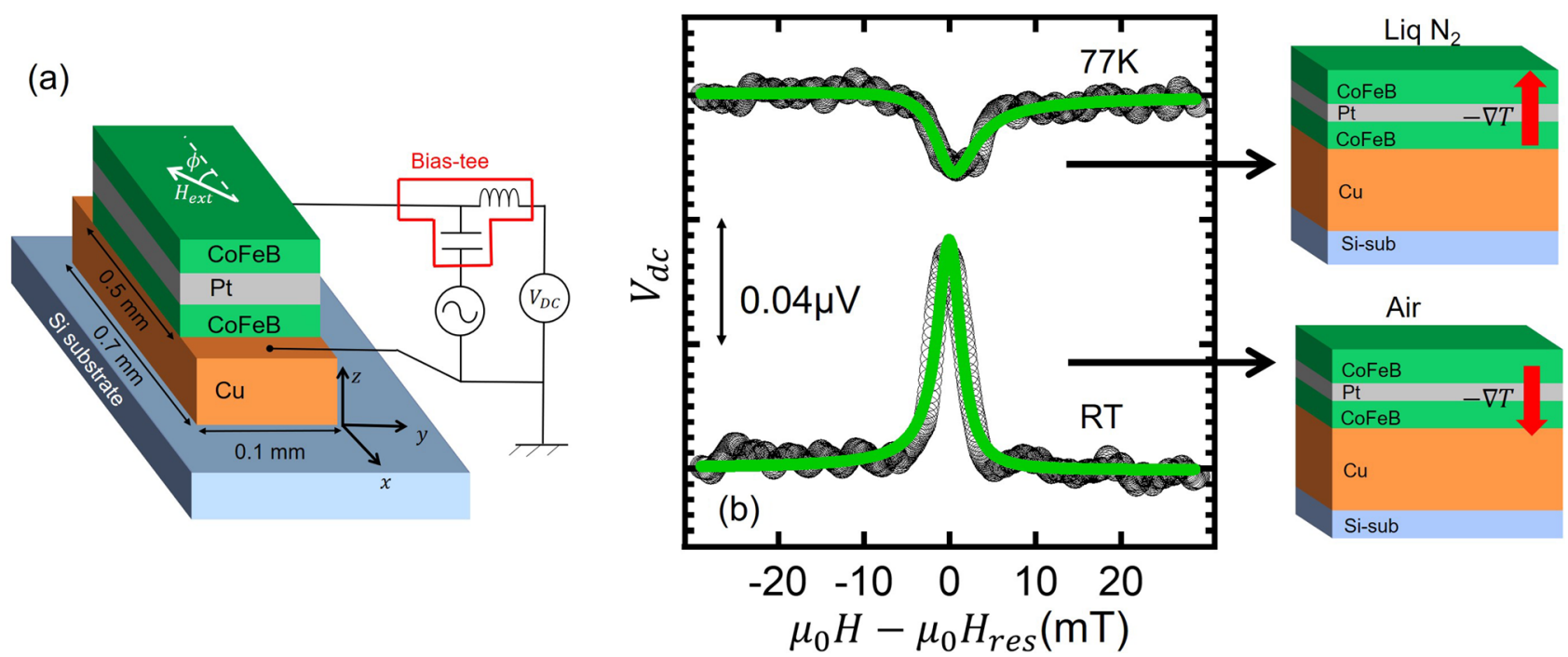

Figure 3. (a) Schematic illustration of the $\mathrm{CoFeB} / \mathrm{Pt} / \mathrm{CoFeB}$ trilayer device fabricated on a $\mathrm{Cu}$ stripline and the probe configuration for the RF measurement. (b) Field dependence of the dc voltage under the microwave with $f=6 \mathrm{GHz}$ measured at room temperature (bottom) and $77 \mathrm{~K}$ (top). The solid lines represent the fitting curve using Eq. (2). The inset show the schematic illustration of the heat flow direction due to the FMR heating for each temperature.

We have also performed a similar measurement at $77 \mathrm{~K}$ by immersing the sample in liquid Nitrogen. In this situation, the temperature of the specimen surface is fixed at the liquid Nitrogen temperature. leading to the acceleration of the upward heat-flow. Therefore, we expect the enhancement of the symmetric voltage with the same sign as that at RT. As can be seen in Fig. 2c, we have observed a clear negative symmetric component with the magnitude larger than that at room temperature (RT). This is a surprising fact because the longitudinal resistance of the sample is much smaller than that at room temperature owing to the reduction of the $\mathrm{Cu}$-electrode resistance. Since the induced voltage is proportional to the longitudinal resistance of the sample, this result indicates the significant enhancement of the thermal spin injection efficiency due to the effective increase of the temperature gradient. We also emphasize that the field dependence is well reproduced by Eq. (2) and that the ratio defined by $V_{S} / V_{A}$ at $77 \mathrm{~K}$ exceeds 50 , much larger than that at room temperature, as shown in Fig. $2 \mathrm{~d}$. This is also consistent with the enhanced efficiency for the dynamical spin injection at $77 \mathrm{~K}$.

To obtain more definite evidence of the significant contribution of the thermal spin injection, we have prepared a similar trilayer on a $\mathrm{Cu}$ strip line, as schematically shown in Fig. 3a. By considering the aforementioned results, the location of the $\mathrm{Cu}$ strip line significantly affects the temperature profile in the trilayer ${ }^{19,24}$. In the present structure, at room temperature, the $\mathrm{Cu}$ heat sink in between the trilayer and the Si substrate increases the downward heat flow, leading to the enhancement of the negative ISHE signal. On the other hand, at $77 \mathrm{~K}$, the upward heat flow decreases, leading to the reduction of the ISHE signal. Figure 3b shows the observed spectra at RT and $77 \mathrm{~K}$ together with their fitting curves using Eq. (2). The results show good agreement with our expectation. These strongly support that the thermal contribution in the dynamical spin injection is important.

It also should be noted that there are two possible contributions for the thermal spin injection. One is the spin-dependent Seebeck effect driven by the spin-polarized current and the other one is the spin Seebeck effect driven by the magnon spin current. In the metallic structure, the spin-dependent Seebeck effect was naively considered as the major mechanism. However, recent studies indicates the importance of the magnon Seebeck effect in the metallic system ${ }^{32,33}$. Since it is still an important milestone to identify the contribution of each component separately, the dynamical spin injection under the temperature control may solve the key for the quantitative analysis of each contribution Thus, the combination between the spin pumping and the thermal spin injection is a key for the efficient dynamical spin injection.

\section{Conclusion}

We have examined the dynamical spin injection in the $\mathrm{CoFeB} / \mathrm{Pt} / \mathrm{CoFeB}$ trilayer by the electrical detection method using a thick $\mathrm{Cu}$ electrode. The efficiency of the dynamical spin injection was significantly reduced because of the symmetric spin pumping from the upper and lower interfaces. However, the finite symmetric component due to the ISHE was found to be induced in the voltage spectra. By changing the temperature gradient in the trilayer, we confirmed that the origin of the ISHE voltage is the thermal spin injection. Our results indicate that the heat control is significant in order to perform the efficient dynamical spin injection.

Received: 25 October 2021; Accepted: 1 February 2022

Published online: 02 March 2022 


\section{References}

1. Wolf, S. A. et al. Spintronics: a spin-based electronics vision for the future. Science (80-.) 294, 1488. https://doi.org/10.1126/scien ce.1065389 (2001).

2. Maekawa, S., Valenzuela, S., Saitoh, E. \& Kimura, T. Spin Current (2012).

3. Slonczewski, J. C. Current-driven excitation of magnetic multilayers. J. Magn. Magn. Mater. 159, L1, ISSN 03048853, http://linki nghub.elsevier.com/retrieve/pii/0304885396000625 (1996).

4. Berger, L. Emission of spin waves by a magnetic multilayer traversed by a current. Phys. Rev. B Condens. Matter Mater. Phys. 54, 9353. https://doi.org/10.1103/PhysRevB.54.9353 (1996).

5. Johnson, M. \& Silsbee, R. H. Spin-injection experiment. Phys. Rev. B 37, 5326. https://doi.org/10.1103/PhysRevB.37.5326 (1988).

6. Jedema, F. J., Filip, A. T. \& Van Wees, B. J. Electrical spin injection and accumulation at room temperature in an all-metal mesoscopic spin valve. Nature 410, 345, ISSN 0028-0836, http://www.nature.com/articles/35066533 (2001).

7. Kimura, T., Hamrle, J., Otani, Y., Tsukagoshi, K. \& Aoyagi, Y. Spin-dependent boundary resistance in the lateral spin-valve structure. Appl. Phys. Lett. 85, 3501. https://doi.org/10.1063/1.1805698 (2004).

8. Jansen, R. Silicon spintronics, www.nature.com/naturematerials (2012).

9. Johnson, M. \& Silsbee, R. H. Thermodynamic analysis of interfacial transport and of the thermomagnetoelectric system. Phys. Rev. B 35, 4959. https://doi.org/10.1103/PhysRevB.35.4959 (1987).

10. Uchida, K. et al. Observation of the spin Seebeck effect. Nature 455, 778. https://doi.org/10.1038/nature07321 (2008).

11. Bauer, G. E. W., Saitoh, E. \& Van Wees, B. J. Spin caloritronics, Nat. Mater. 11, 391, ISSN 14764660, arxiv:1107.4395v1, http://www. ncbi.nlm.nih.gov/pubmed/22522639http://www.nature.com/articles/nmat3301 (2012).

12. Slachter, A., Bakker, F. L., Adam, J.-P. P. \& Van Wees, B. J. Thermally driven spin injection from a ferromagnet into a non-magnetic metal, Nat. Phys. 6, 879, ISSN 17452481, arxiv:1004.1566, http://www.nature.com/doifinder/10.1038/nphys1767http://www.nature. com/articles/nphys1767 (2010).

13. Erekhinsky, M., Casanova, F., Schuller, I. K. \& Sharoni, A. Spin-dependent Seebeck effect in non-local spin valve devices. Appl. Phys. Lett. 100, 212401. https://doi.org/10.1063/1.4717752 (2012).

14. Hu, S., Itoh, H. \& Kimura, T. Efficient thermal spin injection using CoFeAl nanowire. NPG Asia Mater. 6, e127. https://doi.org/ 10.1038/am.2014.74 (2014).

15. Saitoh, E., Ueda, M., Miyajima, H. \& Tatara, G. Conversion of spin current into charge current at room temperature: inverse spinHall effect. Appl. Phys. Lett.https://doi.org/10.1063/1.2199473 (2006).

16. Ando, K. Dynamical generation of spin currents, Semicond. Sci. Technol. 29, 043002, ISSN 0268-1242, http://stacks.iop.org/0268$1242 / 29 / \mathrm{i}=4 / \mathrm{a}=043002$ ?key=crossref.9b3d8f8cbb015b8fd16b1b45267f4562 (2014)

17. Shikoh, E. et al. Spin-Pump-Induced Spin Transport in span class. Phys. Rev. Lett. 110, 127201 (2013).

18. Kuhlmann, N., Swoboda, C., Vogel, A., Matsuyama, T. \& Meier, G. All-metal lateral spin valve operated by spin pumping. Phys. Rev. B Condens. Matter Mater. Phys. 87, 104409. https://doi.org/10.1103/PhysRevB.87.104409 (2013).

19. Yamanoi, K., Yokotani, Y. \& Kimura, T. Dynamical spin injection based on heating effect due to ferromagnetic resonance. Phys. Rev. Appl.https://doi.org/10.1103/PhysRevApplied.8.054031 (2017).

20. Mizukami, S., Ando, Y. \& Miyazaki, T. The study on ferromagnetic resonance linewidth for NM/80NiFe/NM ( NM=Cu, Ta, $\mathrm{Pd}$ and Pt) Films, Jpn. J. Appl. Phys. 40, 580, ISSN 0021-4922, http://stacks.iop.org/1347-4065/40/580 (2001).

21. Tserkovnyak, Y., Brataas, A. \& Bauer, G. E. W. Spin pumping and magnetization dynamics in metallic multilayers. Phys. Rev. Lett. 88, 117601. https://doi.org/10.1103/PhysRevLett.88.117601 (2002).

22. Heinrich, B. et al. Dynamic exchange coupling in magnetic bilayers. Phys. Rev. Lett. 90, 187601. https://doi.org/10.1103/PhysR evLett.90.187601 (2003).

23. Bakker, F. L., Flipse, J., Slachter, A., Wagenaar, D. \& van Wees, B. J. Thermoelectric detection of ferromagnetic resonance of a nanoscale ferromagnet. Phys. Rev. Lett. 108, 167602. https://doi.org/10.1103/PhysRevLett.108.167602 (2012).

24. Yamanoi, K., Yokotani, Y. \& Kimura, T. Heat dissipation due to ferromagnetic resonance in a ferromagnetic metal monitored by electrical resistance measurement. Appl. Phys. Lett. 107, 1 (2015).

25. Cho, S. U. et al. Mechanical signature of heat generated in a current-driven ferromagnetic resonance system. Phys. Rev. Appl.https:// doi.org/10.1103/PhysRevApplied.8.014038 (2017).

26. Lin, W. \& Chien, C. L. arXiv, arxiv:1804.01392 (2018)

27. Obinata, S., Ohnishi, K. \& Kimura, T. Significant suppression of galvanomagnetic signal under dynamical spin injection in CoFeB/ Pt bilayer. Appl. Phys. Lett. 118, 152401. https://doi.org/10.1063/5.0046601 (2021).

28. Obinata, S., Iimori, R., Ohnishi, K. \& Kimura, T. Quantitative evaluation of heating effect on dynamical spin injection using CoFeB/ $\mathrm{Pt} / \mathrm{CoFeB}$ trilayered film. IEEE Trans. Magn. (2021).

29. Bilzer, C. et al. Study of the dynamic magnetic properties of soft CoFeB films. J. Appl. Phys. 100, 053903. https://doi.org/10.1063/1. 2337165 (2006).

30. Kondou, K. et al. Influence of inverse spin Hall effect in spin-torque ferromagnetic resonance measurements. Appl. Phys. Express 9, 023002. https://doi.org/10.7567/APEX.9.023002 (2016).

31. Harder, M., Gui, Y. \& Hu, C. M. Electrical detection of magnetization dynamics via spin rectification effects. Phys. Rep.https://doi. org/10.1016/j.physrep.2016.10.002 (2016).

32. Iguchi, R. et al. Thermographic measurements of spin-current-induced temperature modulation in metallic bilayers. Phys. Rev. B 98, 014402 (2018).

33. Beens, M., Heremans, J. P., Tserkovnyak, Y. \& Duine, R. Magnons versus electrons in thermal spin transport through metallic interfaces. J. Phys. D Appl. Phys. 51, 394002 (2018).

\section{Acknowledgements}

This work is partially supported by Grant-in-Aid for Challenging Research (Pioneering)(17H06227) and JST CREST (JPMJCR18J1).

\section{Author contributions}

S.O. carried out the main part of the experimental work, including the preparation of the sample and wrote the main manuscript text including the figure preparations. R.I. prepared the sample. K.O. and T.K. supervised the experimental research and finalized the manuscript. All authors reviewed the manuscript.

\section{Competing interests}

The authors declare no competing interests.

\section{Additional information}

Correspondence and requests for materials should be addressed to T.K. 
Reprints and permissions information is available at www.nature.com/reprints.

Publisher's note Springer Nature remains neutral with regard to jurisdictional claims in published maps and institutional affiliations.

(c) (i) Open Access This article is licensed under a Creative Commons Attribution 4.0 International License, which permits use, sharing, adaptation, distribution and reproduction in any medium or format, as long as you give appropriate credit to the original author(s) and the source, provide a link to the Creative Commons licence, and indicate if changes were made. The images or other third party material in this article are included in the article's Creative Commons licence, unless indicated otherwise in a credit line to the material. If material is not included in the article's Creative Commons licence and your intended use is not permitted by statutory regulation or exceeds the permitted use, you will need to obtain permission directly from the copyright holder. To view a copy of this licence, visit http://creativecommons.org/licenses/by/4.0/.

(C) The Author(s) 2022 